\title{
Buckling instability in thin soft elliptical particles
}

\author{
N. A. Usov, ${ }^{1,2}$ Ching-Ray Chang, ${ }^{2}$ and Zung-Hang $\mathrm{Wei}^{2}$ \\ ${ }^{1}$ Troitsk Institute for Innovation and Fusion Research, 142190, Troitsk, Moscow region, Russia \\ ${ }^{2}$ Department of Physics, National Taiwan University, Taipei, Taiwan, 10617, Republic of China
}

(Received 16 June 2002; published 27 November 2002)

\begin{abstract}
Buckling instability is proved to be the easiest nucleation mode for an elongated soft elliptical particle with a thickness of the order of the exchange length. The nucleation field saturates as a function of the particle length, and approaches that of a very long bar with a rectangular cross section. The bar nucleation field is estimated theoretically as a function of the bar width, thickness, and material parameters. A step like behavior of nucleation field is revealed by means of a numerical simulation for elliptical particles with a moderate aspect ratio.

DOI: 10.1103/PhysRevB.66.184431

PACS number(s): 75.60.Jk, 75.75.+a
\end{abstract}

\section{INTRODUCTION}

Understanding magnetization reversal in thin ferromagnetic particles and patterned ferromagnetic structures is important both for fundamental magnetism and for various technological applications. ${ }^{1}$ It is found experimentally that there is a difference in magnetization reversal mechanisms for particles of rectangular ${ }^{2-4}$ and smooth elliptical shapes. ${ }^{5,6}$ For soft rectangular particles of submicron sizes there is a closure domain structure near the particles' edges. As a result, for these particles magnetization reversal begins from the end domains. ${ }^{4}$ Also, trapped vortices can cause an anomalous switching between stable magnetization states in rectangular particles. To expel the vortices a large external magnetic field, of the order of the saturation magnetization, is required. ${ }^{3}$

Quite a different scenario of the magnetization reversal was revealed recently in cylindrical and elliptical particles. ${ }^{5-7}$ As numerical simulations showed, ${ }^{7}$ for a soft elliptical particle the range of stability of a quasiuniform magnetization increases considerably with an increase of the particle aspect ratio, $L_{x} / L_{y}>1$. Therefore, after saturation of an elongated submicron particle in a large external magnetic field the quasiuniform state remains stable when the applied field decreases to zero. Magnetization reversal starts at a negative field due to the development of a certain instability mode. The shape of the mode observed, ${ }^{7}$ as shown in Fig. 1, resembles a buckling mode known for an elongated ellipsoidal particle. ${ }^{8}$

According to a numerical simulation carried out in this paper, for an elliptical particle with a moderate aspect ratio $L_{x} / L_{y}<2 \sim 2.5$, the instability mode can be stable in a certain interval of the external magnetic field, though in this interval the mode amplitude increases gradually as a function of the field. Eventually magnetization reversal occurs due to a penetration of several vortices across the particle. On the other hand, for elongated elliptical particles with $L_{x} / L_{y}>3$ the buckling occurs at large enough reversed magnetic field. As a result, there is no interval for mode stability, the hysteresis loop is rectangular, and the vortices can exist only as transient magnetization states. Thus one may hope that the magnetization reversal in an elongated elliptical particle is more stable with respect to shape deviations, the presence of imperfections, etc.

The goal of the present paper is to estimate theoretically the characteristic field for the onset of a buckling instability in a thin elongated elliptical particle. Using the variational approach, ${ }^{8}$ in Sec. II we first calculate the nucleation field for a very long bar with a rectangular cross section. Then this result is generalized to the case of an elongated elliptical particle taking into account a demagnetizing field near the middle part of the particle. ${ }^{6}$ The results of a numerical simulation of the buckling instability in a soft elliptical particle, as a function of the particle aspect ratio, are presented in Sec. III.

\section{BUCKLING INSTABILITY}

Consider a very long ferromagnetic bar with a rectangular cross section with a width $L_{y}=2 a$ and a thickness $L_{z}=2 b$. Due to shape anisotropy the bar, with a large saturation magnetization $M_{s}$ and small magnetic anisotropy constant $K_{1}$, is uniformly magnetized along the $x$ direction, the unit magnetization vector being $\boldsymbol{\alpha}=(1,0,0)$. Let an external uniform magnetic field $H_{0}$ be applied opposite to the bar magnetization. A typical problem of micromagnetics ${ }^{9}$ is to find a critical value of the applied field, the so-called nucleation field $H_{c}$, at which the bar magnetization deviates from the uniform one. It is sufficient to consider a small perturbation of the unit magnetization vector, $\delta \boldsymbol{\alpha}=\left(0, \alpha_{y}, \alpha_{z}\right)$, and minimize its free energy associated with this deviation, ${ }^{9}$

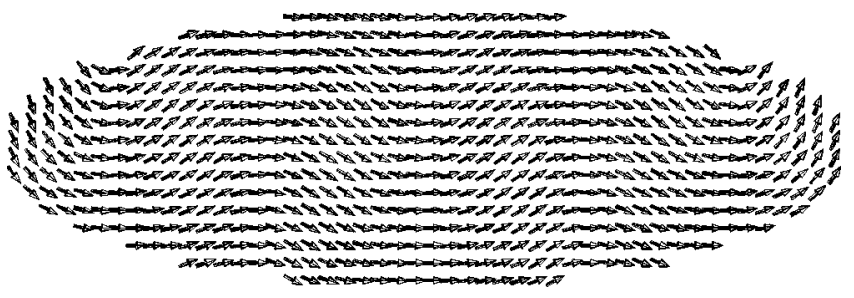

FIG. 1. The buckling spin configuration in an elliptical element under the reversed magnetic field 980 Oe, which is smaller than the switching field 1000 Oe. The dimension of the element is $360 \mathrm{~nm}$ in the long axis, $120 \mathrm{~nm}$ in the short axis, and $30 \mathrm{~nm}$ in thickness, respectively. 


$$
\begin{aligned}
W= & \frac{C}{2} \int d \nu\left[\left(\nabla \alpha_{y}\right)^{2}+\left(\nabla \alpha_{z}\right)^{2}\right] \\
& +\left(K_{1}+\frac{H_{0} M_{s}}{2}\right) \int d \nu\left[\left(\alpha_{y}\right)^{2}+\left(\alpha_{z}\right)^{2}\right]+W_{m},
\end{aligned}
$$

where $C$ is the exchange constant and $W_{m}$ is the magnetostatic energy contribution. The easy anisotropy axis is taken to be parallel to the long side of the bar. In the present paper we are interested in the case of a sufficiently flat bar, $b / a$ $\ll 1$. One can expect that in this case the buckling mode discussed earlier for infinite ferromagnetic cylinder ${ }^{8}$ has the lowest nucleation field. Note that this possibility was not considered in the Refs. 10 and 11.

For a bar with an aspect ratio $b / a \ll 1$ one can neglect the transverse magnetization component $\left|\alpha_{z}\right| \ll\left|\alpha_{y}\right|$. In addition, in the case when the bar thickness $L_{z}$ is of the order of the exchange length $R_{0}=\sqrt{C} / M_{s}$, the $\alpha_{y}$ component does not depend on the $z$ coordinate. Therefore, Brown's equation ${ }^{9}$ for the $a_{y}$ component can be written as

$$
\begin{gathered}
-C\left(\frac{\partial^{2} \alpha_{y}}{\partial x^{2}}+\frac{\partial^{2} \alpha_{y}}{\partial y^{2}}\right)+\left(2 K_{1}+M_{s} H_{0}\right) \alpha_{y}+M_{s} \frac{\partial U}{\partial y}=0, \\
\left.\frac{\partial \alpha_{y}}{\partial y}\right|_{y= \pm a}=0 .
\end{gathered}
$$

Due to the small thickness of the bar, it is sufficient to determine a magnetic potential $U$ near the middle plate of the bar, at $z=0$ only, so that $U=U(x, y, 0)$. One can separate the variables in Eq. (2) setting

$$
\alpha_{y}=\cos (k x) f(y), \quad U=M_{s} \cos (k x) \Phi(y),
$$

where $k$ is the mode wave number and $\Phi(y)$ has an integral representation $^{12}$

$$
\begin{aligned}
\Phi(y)= & \int_{-\infty}^{\infty} d x_{1} \int_{-a}^{a} d y_{1} \int_{-b}^{b} d z_{1} \cos \left(k x_{1}\right) f\left(y_{1}\right) \\
& \times \frac{\partial}{\partial y_{1}} \frac{1}{\left[x_{1}^{2}+\left(y-y_{1}\right)^{2}+z_{1}^{2}\right]^{1 / 2}} .
\end{aligned}
$$

Therefore, the function $f(y)$ satisfies a one-dimensional integrodifferential equation

$$
C\left(-\frac{d^{2} f}{d y^{2}}+k^{2} f\right)+\left(2 K_{1}+M_{s} H_{0}\right) f+M_{s}^{2} \frac{\partial \Phi}{\partial y}=0,
$$

with the boundary condition $d f / d y=0$ at the lateral sides of the bar, $y= \pm a$.

A solution of the boundary value problem [Eq. (5)] can be presented by a Fourier series

$$
f(y)=A\left(1+\sum_{n} a_{n}(k) \cos \left(2 \pi n y / L_{y}\right)\right),
$$

where $A$ is the mode amplitude. The coefficients $a_{n}(k), n$ $=1,2, \ldots$, in Eq. (6) can be estimated by taking into account the magnetostatic energy of surface and volume magnetic charges. In the simplest situation one can neglect the $y$ dependence of the $\alpha_{y}$ component, setting all the coefficients $a_{n}=0$. This means that one takes into account only magnetostatic energy contribution of the surface magnetic charge located at the lateral sides of the bar. Then, the corresponding free energy of the buckling mode is given by ${ }^{13}$

$$
\begin{aligned}
\frac{W(\xi)}{M_{s}^{2} V}= & A^{2}\left(\frac{R_{0}^{2}}{4 L_{y}^{2}} \xi^{2}+\frac{H_{0}}{4 M_{s}}+\frac{K_{1}}{2 M_{s}^{2}}+\frac{2 L_{z}}{L_{y}} \int_{0}^{1} d t(1-t)\right. \\
& \left.\times\left\{K_{0}\left(\xi L_{z} t / L_{y}\right)-K_{0}\left[\xi \sqrt{1+\left(L_{z} t / L_{y}\right)^{2}}\right]\right\}\right)
\end{aligned}
$$

Here $V$ is the bar volume, $\xi=k L_{y}$ is the reduced wave number, and $K_{0}(x)$ is the modified Bessel function. ${ }^{14}$ Using an analytical approximation for the function $K_{0},{ }^{14}$ one can minimize Eq. (7) with respect to $\xi$ numerically. Evidently, the result of minimization depends on two dimensionless parameters, $L_{z} / R_{0}$ and $L_{z} / L_{y}$. The nucleation field for the buckling mode can be obtained then by equating the bracket in the right hand side of Eq. (7) to zero. Actually, the mode amplitude is zero, $A=0$, while this bracket remains positive.

For more accurate estimation of the bar nucleation field the $y$ dependence of the $\alpha_{y}$ component have to be taken into account. It is found that for a bar with moderate aspect ratio, $3<L_{z} / L_{y}<8$, the Fourier series [Eq. (6)] converges sufficiently fast. Therefore, one can take into account the first Fourier coefficient only. The typical value of this coefficient, $a_{1} \approx 0.3-0.4$, accounts for a relative value of the surface magnetic charge scattered into the bar volume. The corresponding correction to the nucleation field is found to be of the order of $15-20 \%$. For a wider bar the Fourier series for function $f(y)$ converges more slowly. In this case the Ritz function

$$
f(y)=1-\gamma\{\exp [\eta(y-L y / 2)]+\exp [-\eta(y+L y / 2)]\}
$$

turns out to be useful. This function describes the layers of the volume magnetic charge with characteristic width $1 / \eta$ $<L_{y}$ located near the lateral sides of the bar. Coefficient $\gamma$ controls the density of the surface magnetic charge. With this function a reduction in the nucleation field of the order of $50 \%$ with respect to the value given by Eq. (7) is obtained for a sufficiently wide bar. ${ }^{15}$

Figure 2(a) shows the reduced nucleation field for the buckling instability as a function of the dimensionless parameters mentioned, the magnetic anisotropy being neglected. The reduced wavelength of the mode, $\lambda / L_{y}$, where $\lambda=2 \pi / k$, is shown in Fig. 2(b). One can see in Fig. 2(a) that at a fixed thickness the absolute value of the nucleation field for the buckling mode decreases considerably as the bar width decreases. When $L_{z} / R_{0}<1$ the wavelength of the mode can be very large and the buckling instability is close to a uniform rotation. On the other hand, it becomes comparable with the bar width if $L_{z} / R_{0} \geqslant 1$.

The latter fact means that in the first approximation one can use a similar approach to estimate the nucleation field of an elongated elliptical particle with sizes $L_{x} \gg L_{y}$ and $L_{z} / R_{0} \geqslant 1$. Actually, the width of the elliptical particle is a 


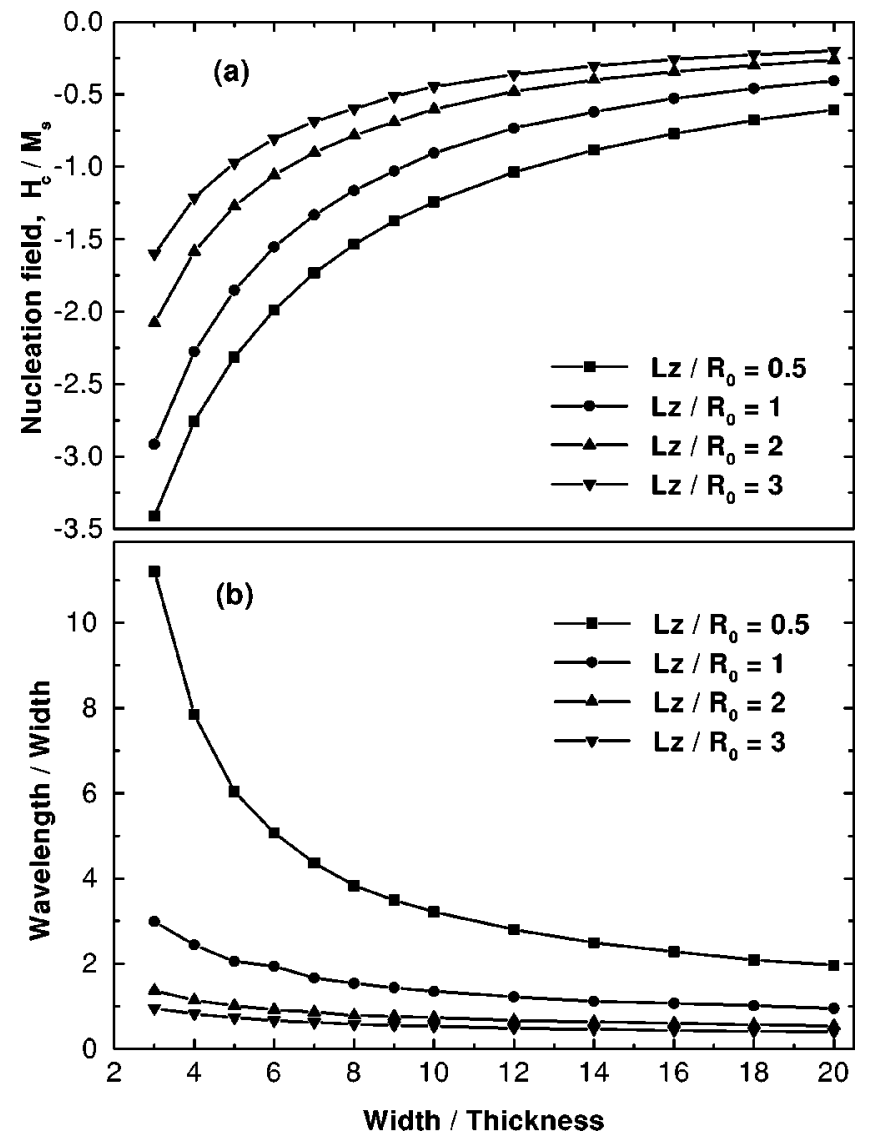

FIG. 2. Nucleation field (a) and wavelength (b) of the buckling instability in a very long bar as a function of the bar aspect ratio.

decreasing function of the longitudinal coordinate, $L_{y}(x)$ $=L_{y}(0)\left[1-\left(2 x / L_{x}\right)^{2}\right]^{1 / 2}$. This function is smooth enough near the middle part of the particle, at $x \approx 0$. Suppose that for an elongated elliptical particle there is a local nucleation field $H_{c}(x)$, which depends on the local width of the particle, $L_{y}(x)$. This suggestion can be justified if the wavelength of the mode is of the order of the particle width, $\lambda \sim L_{y}(0)$ $\ll L_{x}$. In this case the lowest local nucleation field (algebraically) corresponds to the middle part of the elliptical element, $x \approx 0$. On the other hand, in contrast with the bar, a demagnetizing field $H_{d}$ has a nonzero value near the center of a thin $\left(L_{z} \ll L_{x} ; L_{y}\right)$ uniformly magnetized elliptical particle,

$H_{d} \approx-8 M_{s} \frac{L_{y} L_{z}}{L_{x}^{2} p}[K(p)-E(p)], \quad p=1-\left(L_{y} / L_{x}\right)^{2}$,

where $K(p)$ and $E(p)$ are the complete elliptic integrals of the first and second kind, respectively. ${ }^{14}$ Then the nucleation field of an elongated elliptical particle, $H_{c, e l}$, can be estimated using the approximate relation ${ }^{6} H_{c, e l}=H_{c, b a r}-H_{d}$, where $H_{c, b a r}$ is the nucleation field of a very long rectangular bar with the same thickness and width.

\section{NUMERICAL SIMULATION}

The variational estimation of the nucleation field of Permalloy elliptical particles $\left(M_{s}=800 \mathrm{emu} / \mathrm{cm}^{3}, C=2\right.$

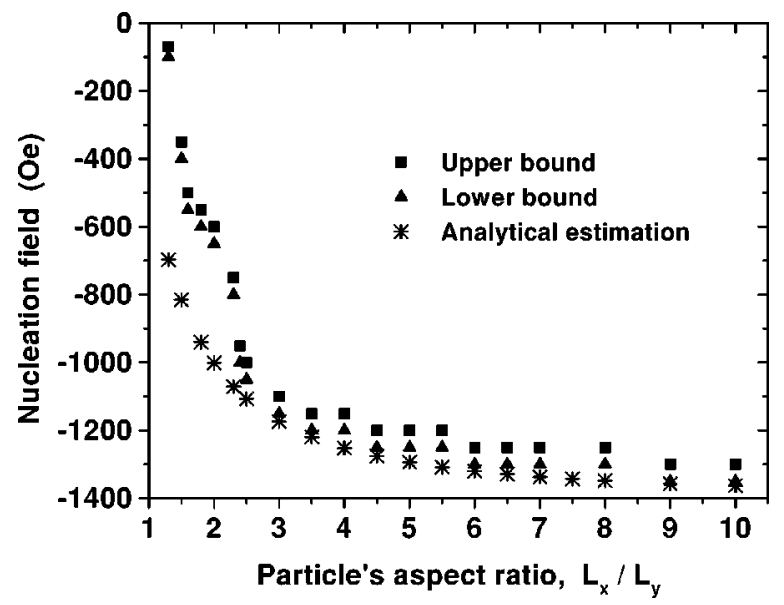

FIG. 3. Analytical estimation of the onset of instability in a soft type elliptical element with a thickness $L_{z}=20 \mathrm{~nm}$ and a width $L_{y}$ $=100 \mathrm{~nm}$ in comparison with the numerical simulation data. The quasiuniform magnetization is certainly stable above the upper bound and unstable below the lower bound.

$\times 10^{-6} \mathrm{erg} / \mathrm{cm}$ ), as a function of the particle length, is given in Fig. 3. To check and validate the assumptions made in Sec. II, a numerical simulation of the magnetization reversal in a flat elliptical particle, with thickness $L_{z}=20 \mathrm{~nm}$ and width $L_{y}=100 \mathrm{~nm}$, is performed for different aspect ratios $1.3 \leqslant L_{x} / L_{y} \leqslant 10$. The numerical simulation data are compared with the variational estimation of the particle nucleation field in Fig. 3. For a numerical simulation an elliptical particle is subdivided into an array of uniformly magnetized cubes of fixed size $l_{0}=5 \mathrm{~nm}$, small enough with respect to the exchange length $R_{0}=\sqrt{C} / M_{s} \approx 18 \mathrm{~nm}$. A direct integration of the Landau-Lifshitz-Gilbert equation is used in the numerical simulations according to the usual scheme. ${ }^{7,16} \mathrm{Nu}$ merically, the nucleation field is determined with accuracy of 50 Oe for elliptical particles with an aspect ratio of $L_{x} / L_{y}$ $\geqslant 3$, when the particle nucleation field is rather high, $H_{c}$ $>1000$ Oe, and with an accuracy of 20-30 Oe for smaller values of the aspect ratio. The upper bound to the nucleation field shown in Fig. 3 corresponds to the value of a reversed external magnetic field when the quasiuniform magnetization of the particle is certainly stable. The lower bound gives the value of the external magnetic field under which the quasiuniform magnetization becomes certainly unstable during the time evolution according to the Landau-Lifshitz-Gilbert equation.

One can see in Fig. 3 that the nucleation field of the elliptical particle estimated numerically saturates as a function of particle aspect ratio, in qualitative agreement with the experimental data. ${ }^{6}$ In addition, the numerical simulation data turn out to be in reasonable agreement with the variational estimation except for the case of small aspect ratios, $L_{x} / L_{y}<2.5$, when the basic assumption $H_{c, e l}=H_{c, b a r}-H_{d}$ can hardly be justified. It is also interesting to note that the numerical data show a steplike behavior of the particle nucleation field. The latter is clearly visible in Fig. 3 at small enough aspect ratios.

Figure 4 shows the shape of the nucleation mode, i.e., the $\alpha_{y}$ component, as a function of the reduced coordinate $x / L_{x}$, 

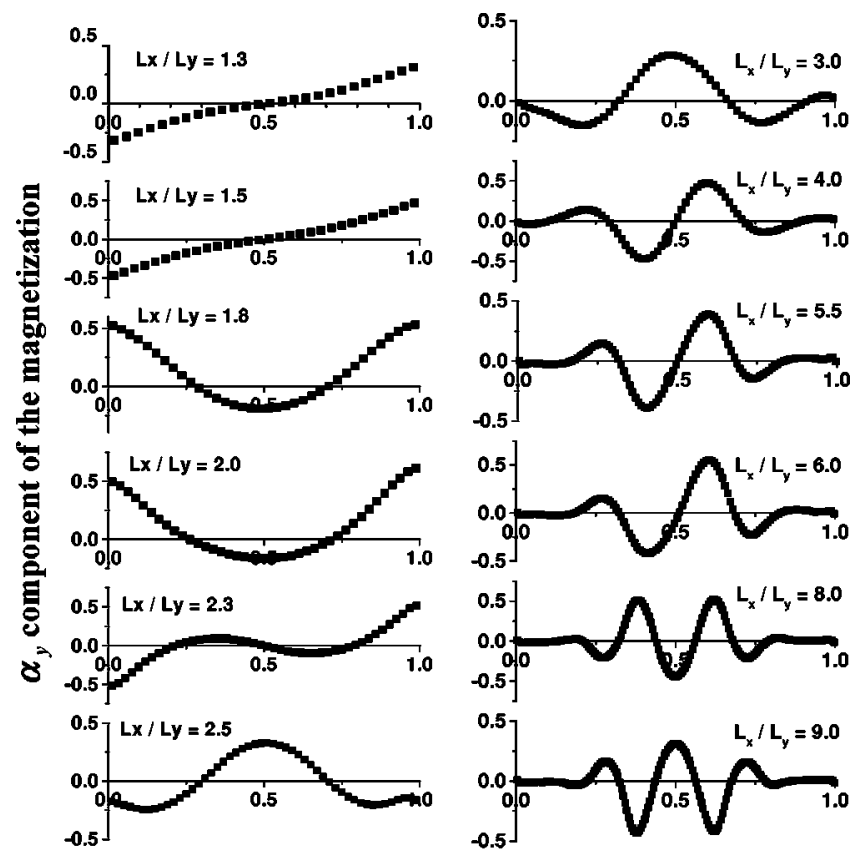

The reduced coordinate, $x / L_{x}$

FIG. 4. The instability mode as a function of the reduced coordinate $x / L_{x}$ for elliptical particles with different aspect ratios. The thickness and width of the particles are the same as in Fig. 3.

along the long particle axis $y=0$, for particles with different aspect ratios. These data are obtained in reversed magnetic fields equal to the corresponding lower bounds to the particle nucleation field shown in Fig. 3. Comparing Figs. 3 and 4 one can see that the steps in the nucleation field shown in Fig. 3 correlate with the abrupt changes in the shape of the nucleation mode as a function of the particle length.

As we mentioned in Sec. I, the mode structures shown in Fig. 4 are stable for small aspect ratios $L_{x} / L_{y}<2.5$. For more elongated particles they are transient pictures only. During the time evolution governed by the Landau-LifshitzGilbert equation the mode amplitude increases gradually, and magnetization reversal eventually occurs due to the penetration of several vortices across the particle.

One can see in Fig. 4 that in accordance with the arguments given in Sec. II, for elongated particles $L_{x} / L_{y} \geqslant 3$, the deviation of the magnetization is maximal at the particle center. Both the period of the oscillations and the nucleation field approach the corresponding values $\lambda / L y \approx 1.8$ and $H_{c, b a r} \approx 1400$ Oe obtained for a long bar with the same width and thickness. However, the amplitude of the oscillations is suppressed considerably close to the particle ends, since elongated particles any magnetization deviation in these regions would lead to a strong in-plane demagnetizing field. Of course, the dependence of the mode amplitude on the $x$ coordinate makes an additional contribution to the exchange energy, given by the first term in Eq. (1), but one can prove that for an elongated particle this contribution is small compared to the total mode free energy. Conversely, as Fig. 4 shows, for particles with an aspect ratio $L_{x} / L_{y} \leqslant 2.5$ the maximal deviation of the magnetization occurs near the particle ends. Also, the onset of the instability deviates considerably from the simple theoretical estimation, and shows a steplike behavior. As we mentioned in Sec. I, for these particles the mode amplitude is stable in some interval of fields after nucleation. The shape of the mode changes considerably with an increase of the particle aspect ratio. It resembles different stable bending states owing to an instability of the quasiuniform magnetization in thin elliptical particles at sufficiently large in-plain dimensions. ${ }^{7,15}$ Clearly, the nucleation of different bending states is responsible for the steplike behavior of the nucleation field in Fig. 3 at small aspect ratios, but the conditions for the bending state nucleation need to be investigated separately.

\section{CONCLUSION}

In summary, magnetization reversal in a soft elliptical particle is studied both numerically and analytically as a function of the particle aspect ratio. It is shown that, unlike particles with a moderate aspect ratio where the maximal deviation of the magnetization occurs near the particle ends, in an elongated elliptical particle the magnetization reversal starts in the middle part of the particle. One can therefore expect that the magnetization reversal in an elongated elliptical particle is more stable with respect to the edge roughness, the presence of imperfections, etc., and, consequently, it is easier to control. It is also found that the nucleation field in an elongated elliptical particle saturates as a function of the particle length, and approaches the nucleation field of a very long bar with the same width and thickness. The bar nucleation field is estimated analytically as a function of the bar width, thickness, and material parameters. Therefore, the results obtained enable one to predict the nucleation field of an elongated elliptical particle with reasonable accuracy.

\section{ACKNOWLEDGMENTS}

This work was supported in part by the Republic of China National Science Council Grant No. NSC 91-2119-M002001 and Technology Development Program for Academia Grant No. 91-EC-17-A-08-S1-0006.
${ }^{1}$ G.A. Prinz, Science 282, 1660 (1998).

${ }^{2}$ K.J. Kirk, J.N. Chapman, and C.D.W. Wilkinson, Appl. Phys. Lett. 71, 539 (1997).

${ }^{3}$ Jing Shi, S. Tehrani, T. Zhu, Y.F. Zheng, and J.-G. Zhu, Appl. Phys. Lett. 74, 2525 (1999).
${ }^{4}$ K.J. Kirk, M.R. Scheinfein, J.N. Chapman, S. McVitie, M.F. Gillies, B.R. Ward, and J.G. Tennant, J. Phys. D 34, 160 (2001).

${ }^{5}$ R.P. Cowburn, D.K. Koltsov, A.O. Adeyeye, M.E. Welland, and D.M. Tricker, Phys. Rev. Lett. 83, 1042 (1999).

${ }^{6}$ J.A. Johnson, M. Grimsditch, V. Metlushko, P. Vavassori, B. Ilic, 
P Neuzil, and R. Kumar, Appl. Phys. Lett. 77, 4410 (2000).

${ }^{7}$ N.A. Usov, Ching-Ray Chang, and Zung-Hang Wei, J. Appl. Phys. 89, 7591 (2001).

${ }^{8}$ E.H. Frei, S. Shtrikman, and D. Treves, Phys. Rev. 106, 446 (1957).

${ }^{9}$ W.F. Brown, Jr., Micromagnetics (Interscience, New York, 1963).

${ }^{10}$ A. Aharoni, J. Appl. Phys. 34, 2434 (1963).

${ }^{11}$ W.F. Brown, Jr., J. Appl. Phys. 35, 2102 (1964).

${ }^{12}$ A.I. Akhiezer, V.G. Bar'yakhtar, and S.V. Peletminskii, Spin Waves (Wiley, New York, 1968).
${ }^{13}$ Note that an earlier estimation of the surface magnetic charge energy [S.W. Yuan, H.N. Bertram, J.F. Smyth, and S. Schultz, IEEE Trans. Magn. 28, 3171 (1992)] gives approximately twice as low a value as the correct result [Eq. (2)].

${ }^{14}$ Handbook of Mathematical Functions, edited by M. Abramowitz and I.A. Stegun (Dover, New York, 1970).

${ }^{15}$ N.A. Usov, Ching-Ray Chang, and Zung-Hang Wei, J. Appl. Phys. (to be published).

${ }^{16}$ N.A. Usov and S.E. Peschany, J. Magn. Magn. Mater. 130, 275 (1994). 